\title{
Impact of Early Postoperative Enteral Feeding on Hospital Length of Stay in Patients Undergoing Colonic Surgery: Results of a Prospective Randomized Trial
}

\author{
Yves Bendavid, Karyne Martel, Lucas Sideris, Pierre Drolet, Pierre Dubé \\ Departmentof Surgery, Hôpital Maisonneuve-Rosemont, Montréal, Canada \\ Email: bendavid@videotron.ca
}

Received September 13, 2012; revised October 11, 2012; accepted October 20, 2012

\begin{abstract}
Introduction: Early feeding within 24 hours of intestinal surgery seems advantageous in terms of reduction of wound infection, pneumonia and length of hospital stay. The aim of the study is to evaluate the impact of early enteral nutrition in length of hospital stay in comparison to traditional postoperative feeding regimen. Method: This prospective study enrolled 95 patients randomized in two groups: control group patients receive enteral feeding in absence of nausea or vomiting, abdominal distension and after passage of flatus or stools, while patients in experimental group were fed a liquid diet within 12 hours of surgery, followed by a regular diet at the next meal. The primary endpoint was the impact of early oral feeding on hospital length of stay. The secondary endpoint was to measure the impact of the diet reintroduction modality on the incidence of early postoperative morbidity and return of bowel function. Result: Length of hospital stay was slightly diminished in the experimental group compared to control $(8.78 \pm 3.85$ versus $9.41 \pm 5.22)$, but the difference was not statistically significant. Postoperative nausea and vomiting were reported in $24(51.0 \%)$ patients in experimental group and $30(62.5 \%)$ in control group. Only one patient required nasogastric tube insertion. The majority of patients did not demonstrate any postoperative morbidity in both groups. Conclusion: Early enteral nutrition is safe after intestinal surgery. However we did not demonstrate that early enteral feeding diminished length of hospital stay or hastened the return of bowel function.
\end{abstract}

Keywords: Early Enteral Nutrition; Colectomy; Hospital Length of Stay; Randomized Trial

\section{Introduction}

Early enteral nutrition is a novel postoperative approach that consists of any caloric intake introduced within 24 hours of gastrointestinal surgery. Although sometimes regarded as controversial, early enteral nutrition is based on clinical and biological evidence. Animal models demonstrated that early nutrition favors the anastomotic healing in the presence of abdominal sepsis [1]. TNF $\alpha$ increases collagenase activity and decreases collagen synthesis, thus preventing wound healing [2]. In contrast, rats fed within 24 hours presented lower TNF $\alpha$ level and higher anastomotic burst pressure in comparison with animals treated with water [2]. When clinicians started to use this protocol, it was hypothesized that only patients undergoing laparoscopy surgery would benefit from early nutrition, but not those undergoing standard open procedures. However, some trials showed that early enteral feeding was as well tolerated in both open and laparoscopic groups, showing no difference in terms of nasogastric tube insertion and frequency of vomiting, thus emphasizing the high potential of early feeding [3].
Several studies have demonstrated the tolerability and safety of early enteral nutrition in the post operative period when compared to the classic diet reintroduction surgical habit [4]. DiFronzo et al. placed 87 elderly patients undergoing open colon resection on an early postoperative feeding protocol consisting of fluid intake started in the evening of the second postoperative day followed by regular diet in postoperative day 3 [5]. Patients had low morbidity rates and shorter hospital stay [5]. Clinical benefits of early enteral nutrition have now been summarized in a meta-analysis review by Andersen and colleagues [6]. Thirteen randomized trials comparing early enteral feeding to conventional reintroduction of diet after digestive surgery were compiled. Wound infection, pneumonia and mortality were decreased in treated patients versus controls. The diminished mortality results are however controversial as they were mostly reported from the smaller studies or studies of lesser methodological quality [7]. In this same meta- analysis the hospital length of stay (LOS) appeared reduced in 9 of 13 trials. The decrease of hospital length of stay has important 
implications in the short-term quality of life of patients as well as from an economic point of view.

The present study is a prospective randomized trial conducted in a public institution in the Canadian social healthcare system, where the cost of hospitalization and surgery is not an issue for patients. The aim of this study was to assess whether early return to enteral feeding after colonic surgery with restoration of bowel continuity effectively shortened the length of hospital stay, when compared to a more traditional approach of managing diet in the postoperative period.

\section{Material and Methods}

A prospective block randomized controlled trial design was used to determine whether or not early postoperative enteral feeding would result in a significantly shorter LOS in patients undergoing a colonic surgery. Our Institutional review board approved the study. To be included in the study, patients had to be consenting adults with ASA class 1, 2 or 3 physical statuses. Patients had to be scheduled for elective or urgent colectomy with reanastomosis or colostomy closure, by open or laparoscopic approach. ASA class 4 or 5 status, pregnancy, preoperative diagnosis of digestive dismotility disorder, intrabdominal sepsis within 10 days from surgery, chemotherapy within 4 weeks from surgery, radiotherapy in the region where the construction of the anastomosis was planned, intraoperative enterotomy, or concomitant creation of a small bowel anastomosis were considered as exclusion criteria. Eligible patients were identified within three months from their date of surgery by surgeons and research personnel. Demographic and medical characteristics for each patient were collected in a confidential database. Both groups were prepared for surgery in the same fashion through medical evaluation and work up. Bowel preparation was left to the surgeon's discretion and standard antibioprophylaxis and thromboprophylaxis were administered at the time of surgery. There was no specific intervention or protocol in intraoperative and postoperative anesthetic care, but analgesic modality and use of antiemetics was documented. Standardized postoperative sheets containing all pertinent data for the study were filled every day by the surgical team and compiled by dedicated research personnel. Diet tolerance, nausea, vomiting, return to fasting state or reinsertion of nasogastric tube were documented for each patient. Reinsertion of nasogastric tube was performed under the following conditions: failure of standard of care antiemetic regimen for 12 hours or more, significant gastric distension causing vasovagal symptoms, respiratory difficulties, patient discomfort or if otherwise deemed necessary by the surgical team.

Three conditions needed to be fulfilled for control group patients to resume enteral feeding: absence of nausea or vomiting, absence of abdominal distension and passage of flatus or stools. Patients in the experimental group were fed a liquid diet within 12 hours of surgery, followed by a regular diet at the next meal. The primary endpoint was to measure the impact of early oral feeding on hospital length of stay. Other major outcomes included return of digestive motility and tolerance of a diet. The impact of diet reintroduction modality on the incidence of early postoperative complications was also evaluated. Continuous data were compared with the unpaired $t$ test. Categorical data were compared with either the Chi-square test or Fisher's exact test. The return to sustained oral feeding is reported with Kaplan-Meier curves. It was analyzed with the log-rank test (Prism 5, Graphpad Software, La Jolla, CA), a P $<0.05$ was considered significant. Establishing that a difference of one day of hospitalization was considered significant, we calculated that a sample size of 800 patients ( 400 patients per arm) would be necessary. Unfortunately, recruitment was slower than anticipated and, after 45 months, we decided to interrupt the study and analyzed the data from the 95 patients who were enrolled. A retrospective power analysis showed that the study ended up being appropriate to detect a 2.6 days difference in LOS between groups with alpha and beta errors of 0.05 and 0.2 respectively (StatMate 2.0 Graphpad Software, La Jolla, CA).

\section{Results}

Between March 2006 and December 2009, 95 patients were enrolled in the study. Forty-seven patients were assigned to the early enteral feeding (EEF) group, and 48 patients to the control group. Demographic characteristics of patients included in this randomized study are summarized in Table 1. Mean age was 69 years for both arms of the study. Women represented $44.8 \%$ of experimental and $54.1 \%$ of control group. Ten smokers were included into experimental group and 14 into control group. Patients underwent various open or laparoscopic interventions. Epidural analgesia, patient-controlled analgesia (PCA) or subcutaneous opiates were offered in combination with non-steroid anti-inflammatory drugs (NSAIDs) and were administered to all patients included in the study.

As reported in Table 2, no difference was found between groups with regard to LOS, time to first flatus, time to first bowel movement and time to definitive reintroduction of diet. The proportion of patients unable to tolerated reintroduction of diet vs. time is shown in Figure 1. Secondary outcomes of the study are summarized in Table 3. Postoperative nausea and vomiting were reported in $24(51.0 \%)$ patients in the experimental group and $30(62.5 \%)$ in the control group. Thus, anti-emetic 
drugs were administered to $55.3 \%$ of patients in the experimental group and $64.8 \%$ of control group. Only one patient required nasogastric tube insertion. Postoperative complications are also summarized in Table 3 . The majority of patients did not have any clinical adverse event.

Table 1. Emographic characteristics of patients and type of intervention. Data are reported as $\mathbf{n}$ or means \pm SD. There is no significant difference between groups regarding any of these characteristics.

\begin{tabular}{|c|c|c|}
\hline & $\begin{array}{c}\text { Experimental } \\
\text { Group }(\mathrm{n}=47)\end{array}$ & Control Group $(n=48)$ \\
\hline Age (Years) & $69 \pm 9$ & $69 \pm 12$ \\
\hline Women (n) & 21 & 26 \\
\hline $\begin{array}{c}\text { Preoperative } \\
\text { Albuminemia (g/liter) }\end{array}$ & $38.6 \pm 13.8$ & $38.3 \pm 14.5$ \\
\hline Weight $(\mathrm{Kg})$ & $73.2 \pm 14.0$ & $73.3 \pm 17.5$ \\
\hline \multicolumn{3}{|c|}{ Type of Intervention (n) } \\
\hline Right Hemicolectomy & 17 & 20 \\
\hline $\begin{array}{l}\text { Extended Right } \\
\text { Hemicolectomy }\end{array}$ & 1 & 0 \\
\hline Left Colectomy & 4 & 2 \\
\hline Sigmoidectomy & 4 & 2 \\
\hline Anterior Resection & 11 & 10 \\
\hline Low Anterior Resection & 5 & 6 \\
\hline Ileo-Colic Resection & 0 & 1 \\
\hline Subtotal Colectomy & 0 & 2 \\
\hline Total Colectomy & 1 & 0 \\
\hline Transverse Colectomy & 1 & 2 \\
\hline Hartmann's Closure & 3 & 3 \\
\hline \multicolumn{3}{|c|}{ Surgical Approach } \\
\hline Open & 26 & 31 \\
\hline Laparoscopy & 19 & 11 \\
\hline Combined & 2 & 6 \\
\hline \multicolumn{3}{|c|}{ Anastomosis } \\
\hline Manual & 15 & 17 \\
\hline Mechanical & 32 & 31 \\
\hline \multicolumn{3}{|c|}{ Postoperative Analgesia } \\
\hline Epidural & 26 & 30 \\
\hline Sub-Cutaneous Opiates & 15 & 7 \\
\hline $\begin{array}{l}\text { Patient Controlled } \\
\text { Anesthesia }\end{array}$ & 6 & 10 \\
\hline $\mathrm{PO}$ & 0 & 1 \\
\hline
\end{tabular}

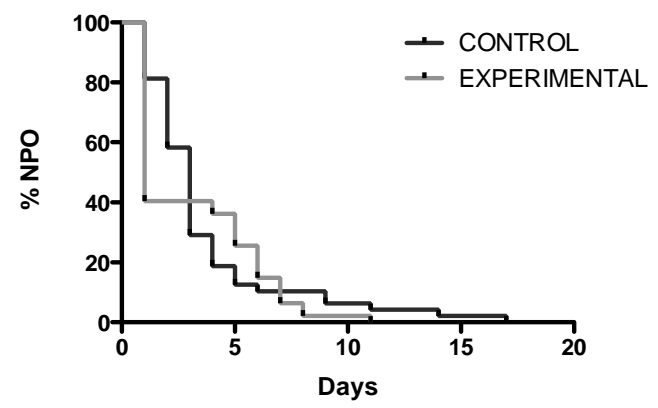

Figure 1. Comparison between groups regarding the percentage of patients unable to tolerate a diet vs time in the postoperative period ( $P$ : 0.56$)$.

Table 2. Primary outcome and related data. Values are reported as means \pm SD and $(95 \% \mathrm{CI})$. No significant difference was observed.

\begin{tabular}{ccccc}
\hline & $\begin{array}{c}\text { Experimental Control Group } \\
\text { Group (n=47) }\end{array}$ & $\begin{array}{c}\text { Difference } \\
\text { between Mean } \\
(\mathrm{n}=48)\end{array}$ & $\begin{array}{c}\mathrm{P} \\
\text { Value }\end{array}$ \\
\hline $\begin{array}{c}\text { Length of } \\
\text { Hospital Stay } \\
\text { (days) }\end{array}$ & $8.7 \pm 3.85$ & $9.4 \pm 5.22$ & $\begin{array}{c}0.6 \\
(-1.2 \text { to } 2.5)\end{array}$ & 0.51 \\
$\begin{array}{c}\text { First Flatus } \\
\text { (days) }\end{array}$ & $2.1 \pm 1.2$ & $2.6 \pm 1.7$ & $\begin{array}{c}0.4 \\
(-1.1 \text { to } 1.1)\end{array}$ & 0.11 \\
$\begin{array}{c}\text { First Bowel } \\
\text { Movement (days) } \\
\text { Definitive }\end{array}$ & $3.5 \pm 1.9$ & $3.5 \pm 1.8$ & $\begin{array}{c}0.0 \\
(-0.7 \text { to } 0.8)\end{array}$ & 0.97 \\
$\begin{array}{c}\text { Reintroduction of } \\
\text { Normal Diet } \\
\text { (days) }\end{array}$ & $3.1 \pm 2.7$ & $3.6 \pm 3.2$ & $\begin{array}{c}0.5 \\
(-0.7 \text { to } 1.8)\end{array}$ & 0.40 \\
\hline
\end{tabular}

\section{Discussion}

Early enteral nutrition after colorectal surgery is becoming an accepted approach. Like other groups, we report no increase in complication rate or their nature with early feeding, and we believe that both approaches are as safe. Most studies do not show a difference in length of hospital stay between early and conventional reintroduction of diet and, not surprisingly, cumulation of data of the thirteen trials included in the meta-analysis by Andersen et al. [6] reflect this by not showing a statistically or clinically significant difference between these two groups. Our results are in accordance with these other studies, but like many others, our trial suffers from being underpowered. Pre-trial statistical power calculations indicated that 385 patients were needed in each group to be able to demonstrate a 1 day difference in hospital stay. During the 3 years of the study, only 95 patients were recruited, and retrospective power studies showed that a difference of 2.6 days $(\alpha=0.05, \beta=0.2)$ in hospital length of stay and $1.7(\alpha=0.05, \beta=0.2)$ days in definitive return to normal diet could have been detected with our sample size. Poor preoperative identification and referral of potential study candidates seem to have been the major 
Table 3. Secondary outcomes.

\begin{tabular}{|c|c|c|}
\hline & $\begin{array}{l}\text { Experimental } \\
\text { Group }(n=47)\end{array}$ & $\begin{array}{l}\text { Control Group } \\
\quad(n=48)\end{array}$ \\
\hline $\begin{array}{c}\text { Postoperative Nausea and } \\
\text { Vomiting }\end{array}$ & 24 & 30 \\
\hline $\begin{array}{c}\text { Postoperative Anti-Emetic Drug } \\
\text { Administration }\end{array}$ & 26 & 31 \\
\hline \multicolumn{3}{|l|}{ Postoperative Complication } \\
\hline None & 36 & 39 \\
\hline Anemia, Transfusion & 0 & 1 \\
\hline Arrhythmia & 1 & 0 \\
\hline Pulmonary Atelectasis & 0 & 1 \\
\hline C. Difficile Positive & 0 & 1 \\
\hline Cellulitis & 1 & 0 \\
\hline Anastomic Dehiscence & 2 & 0 \\
\hline Aponevrotic Dehiscence & 0 & 1 \\
\hline Myocardial Infarction & 1 & 0 \\
\hline Surgical Site Infection & 3 & 2 \\
\hline Pneumonia & 0 & 1 \\
\hline Urinary Tract Infection & 1 & 0 \\
\hline $\begin{array}{l}\text { Urinary and Surgical Site } \\
\text { Infection }\end{array}$ & 0 & 1 \\
\hline Respiratory Failure (Pneumonia) & 0 & 1 \\
\hline Other $^{*}$ & 2 & 1 \\
\hline
\end{tabular}

*Other complications include: intra-abdominal hemorrhage, repeat surgery, pleural effusion necessitating chest tube insertion.

cause for this, as our hospital is a fairly large volume center for colorectal surgery.

The fact that an early feeding regimen was applied to the experimental group did not result in a faster definitive return to diet, and this may also have impacted on the results for length of stay. Indeed, if patients were not able to tolerate diet earlier in the experimental group, there is no reason to believe they should leave the hospital faster than the control group, all other factors being rendered comparable through randomization. Contrarily to multimodality true fast track surgery [8], our study focused on enteral feeding alone and all other parameters were left to surgical and anesthetic discretion. This might also have negatively influenced our results.

Our data also indicates that patients remain in hospital approximately 5 days after they are able to tolerate diet. This might well signify that, in our environment, return of bowel function might not be the main determinant of hospital stay, and this is also found in other studies [9]. Patient comorbidities, their capacity to return home or our efficacy in organizing a transfer to a convalescence institution might at this point be the factors that prolong hospital stay. Additionally, medical care and hospital stay being free for patients in Canada, it might be hypothesized that some patients lose the financial incentive to leave the hospital as soon as possible after surgery to limit cost, thereby prolonging hospital length of stay.

Finally early enteral feeding was applied to all patients that were randomized to the experimental group. To our knowledge no study has investigated the hypothesis that early enteral feeding or fast track protocols should only be utilized for selected patients that are more susceptible to respond well to such approaches. Although all patients should receive appropriate, standard of care medical treatments for their conditions, perhaps patient selection for early enteral feeding and fast track protocols would yield greater results in a selected group of patients, and would provide a greater return on investment from a social point of view, in the context of ever increasing costs of medical care. In summary we believe that early enteral nutrition is not detrimental to patients, but that it is only one of the conditions required to reduce hospital stay and lessen the physiologic impact of major surgery on patients.

\section{REFERENCES}

[1] T. Kiyama, M. Onda, A. Tokunaga, T. Yoshiyuki and A. Barbul, "Effect of Early Postoperative Feeding on the Healing of Colonic Anastomoses in the Presence of Intra-Abdominal Sepsis in Rats," Diseases of the Colon \& Rectum, Vol. 43, No. S10, 2000, pp. S54-S58. doi:10.1007/BF02237227

[2] T. M. Khalili, R. A. Navarro, Y. Middleton and D. R. Margulies, "Early Postoperative Enteral Feeding Increases Anastomotic Strength in a Peritonitis Model," Association for Jewish Studies, Vol. 182, No. 6, 2001, pp. 621-624.

[3] H. Ortiz, P. Armendariz and C. Yarnoz, "Early Postoperative Feeding after Elective Colorectal Surgery Is Not a Benefit Unique to Laparoscopy-Assisted Procedures," International Journal of Colorectal Disease, Vol. 11, No. 5, 1996, pp. 246-249. doi:10.1007/s003840050055

[4] P. Reissman, T. A. Teoh, S. M. Cohen, E. G. Weiss, J. J. Nogueras and S. D. Wexner, "Is Early Oral Feeding Safe after Elective Colorectal Surgery? A Prospective Randomized Trial," Annals of Surgery, Vol. 222, No. 1, 1995, pp. 73-77. doi:10.1097/00000658-199507000-00012

[5] L. A. DiFronzo, N. Yamin, K. Patel and T. X. O'Connell, "Benefits of Early Feeding and Early Hospital Discharge in Elderly Patients Undergoing Open Colon Resection," Journal of the American College of Surgeons, Vol. 197, No. 5, 2003, pp. 747-752. doi:10.1016/S1072-7515(03)00794-4

[6] H. K. Andersen, S. J. Lewis and S. Thomas, "Early Enteral Nutrition within $24 \mathrm{~h}$ of Colorectal Surgery versus Later Commencement of Feeding for Postoperative Complications," Cochrane Database of Systematic Reviews, 
Vol. 18, No. 4, 2006, pp. 1-32.

[7] R. L. Koretz, "Enteral Nutrition: A Hard Look at Some Soft Evidence," Nutrition in Clinical Practice, Vol. 24, No. 3, 2009, pp. 316-324.

doi: $10.1177 / 0884533609335378$

[8] H. Kehlet and D. W. Wilmore, "Multimodal Strategies to Improve Surgical Outcome," Association for Jewish Stu- dies, Vol. 183, No. 6, 2002, pp. 630-641.

[9] Z. Serclová, P. Dytrych, J. Marvan, K. Nová, Z. Hankeová, O. Ryska, et al., "Fast-Track in Open Intestinal Surgery: Prospective Randomized Study," Clinical Nutrition, Vol. 28, No. 6, 2009, pp. 618-624. doi:10.1016/j.clnu.2009.05.009 